\title{
Electric motor for brakes - optimal design
}

\section{Electric Motor for Brakes - Optimal Design}

\author{
Antonino Di Gerlando, Massimiliano Gobbi, and Giampiero Mastinu Politecnico di Milano \\ Alessio Miotto Brembo Spa
}

Citation: Di Gerlando, A., Gobbi, M., Mastinu, G., and Miotto, A., "Electric Motor for Brakes - Optimal Design," SAE Technical Paper 2020-01-0919, 2020, doi:10.4271/2020-01-0919.

\begin{abstract}
A multi-objective optimal design of a brushless DC electric motor for a brake system application is presented. Fifteen design variables are considered for the definition of the stator and rotor geometry, pole pieces and permanent magnets included. Target performance indices (peak torque, efficiency, rotor mass and inertia) are defined together with design constraints that refer to components stress levels and temperature thresholds, not to be surpassed after heavy duty cycles. The mathematical models used for optimization refer to electromagnetic field and related currents computation, to thermo-fluid dynamic simulation, to local stress and vibration assessment. An Artificial Neural Network model, trained with an iterative procedure, is employed for global approximation purposes. This allows to reduce the number of simulation runs needed to find the optimal configurations. Some of the Pareto-optimal solutions resulting from the optimal design process are analysed. They show high improvements of the performance indices with respect to a reference solution.
\end{abstract}

\section{Introduction}

The paper deals with the design of brushless DC electric motors used in a brake-by-wire systems. The clamping force is provided by the electromechanical actuators (EMAs) instead of hydraulic pistons [1]. Typically, while designing a DC brushless electric motor [2], a number of parameters has to be selected, i.e. the slot/pole combination, the stator outer radius, the machine topology, the number of windings, the stator flux density and the choice of materials. A basic outline of a standard methodology is given in $[3,4]$.

In magnetism, shape optimal design problems, i.e. identifying the geometry of the device that is able to provide the required performance while satisfying a set of constraints, have been studied by many authors [6].

A systematic investigation of the multidimensional domain of variation of the design variables can be completed only by using uniformly distributed sequences, as described in [25].

From the viewpoint of the methods suitable to solve multi-objective optimisation problems, the use of approximation techniques to identify response surfaces representing the dependence of the objective functions and constraints on the design variables is extensively applied when time consuming physical models are considered [9]. Globaloptimisation algorithms or evolutionary algorithms, are then applied to find an approximation of the Pareto set [5]. The feasible Pareto optimal solutions can be determined by resorting to the approximated mathematical model and finally validated through the computationally expensive physical simulations.

Page 1 of 6
The paper is structured as follows. In the first section, the brushless DC motor to be designed is presented. The multi-physic modelling of the motor is described in section two. Then the multi-objective optimisation method is introduced including the global approximation approach. The design process results are discussed in the final part of the paper.

\section{Brushless DC motor description (BLDC)}

In Fig. 1. the electro-actuated brake calliper that has been considered is shown. The calliper, in red color is floating. The electric motor is indicated by the arrow. The motor is connected to the calliper by a reduction gearbox.

The brushless DC motor has been designed. Motor topology (stator and rotor) has to be selected according to dimensional constraints in order to fulfil calliper available room. In order to achieve maximum admissible performance, the rotor magnetic field is generated by surface-mounted permanent magnets (PM) and concentrated stator winding.

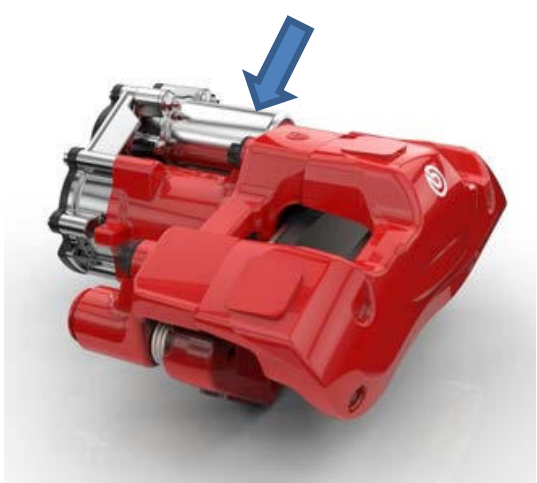

Figure 1. BLDC electric motor integrated into the brake calliper (courtesy of Brembo S.p.A.).

Permanent magnets located on the surface of the rotor (see Fig. 2) are very effective in producing high values of torque. The fixing of the permanent magnets glued onto the rotor surface requires the use of bandages or retaining sleeves due to the centrifugal forces; a metallic sleeve (non-magnetic steel, titanium, ....) or non-metallic bandage (carbon fiber, fiberglass, ....) can be adopted [26]. 
The number of poles and slots of the motor has been selected to fulfil some design constraints (the number of poles must be an even number, the number of slots a multiple of the number of phases) and to maximise the winding factor. The number of poles can't be equal to the number of slots, since this would lead to undesired cogging torque [27]. Cogging torque produces vibrations on the mechanical components of the vehicle so it is necessary to keep it under a determined threshold.

The material used for the magnets is neodymium iron boron $(\mathrm{NdFeB})$ that, compared to the classic alternative of samarium cobalt $(\mathrm{SmCo})$, is more performant, having higher magnetic strength but lower Curie point.

The motor is fed by a $12 \mathrm{~V}$ battery. The current input of the 3 phases is defined by the BCU (Brake Control Unit) model that considers the switch and diode characteristics [20]. The actual simulation condition is with constant current value that allows shorter computational times. The electric motor is tested at the maximum operative temperature required by the manufacturer, that is $120^{\circ} \mathrm{C}$.

The windings section geometry and placement is defined by using the copper slot fill factor, i.e. the ratio between the total section area occupied by the winding conductors over the total available slot section area. The copper slot fill factor has been selected for the reference motor design by finding a reasonable trade-off against winding losses; this value will be kept constant in the optimisation process presented in the next sections. The windings pattern through the slots of the stator is fixed for all the motor configurations and equal to the reference design.

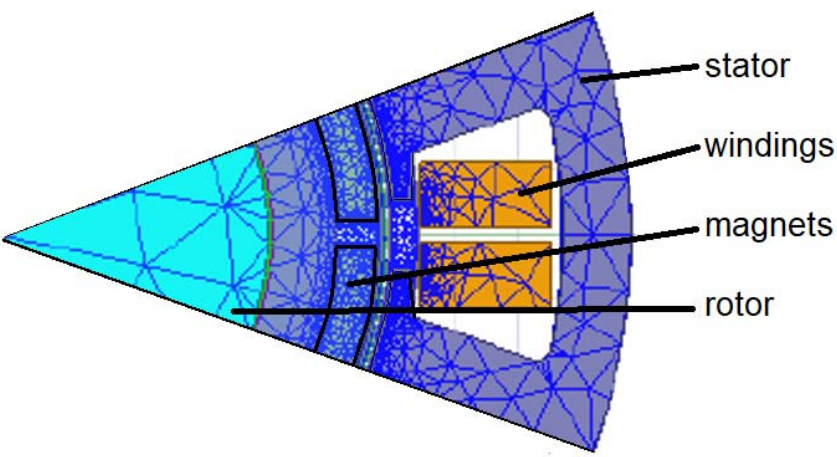

Figure 2. BLDC electric motor, one slot section (preliminary design).

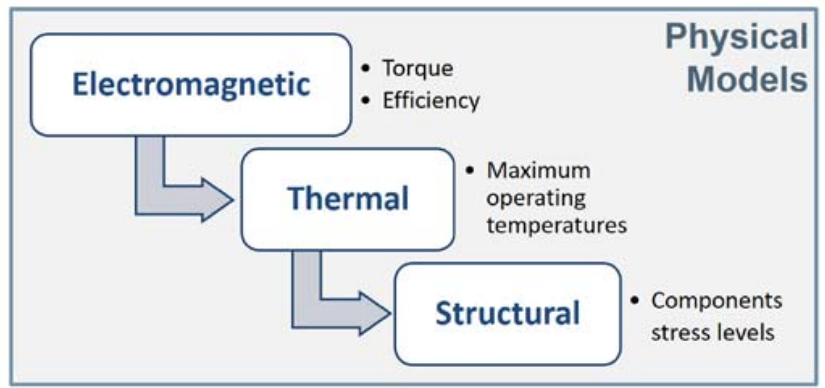

Figure 3.Physical models.

\section{Physical models}

The multi-physics mathematical models of the motor refer to electromagnetic field and related currents computation, to thermo-fluid dynamic simulation, to local stress and vibration assessment (see Fig. 3).

The electromagnetic model is a two-dimensional FE model developed in Motor-CAD®. It can compute the maximum output torque, the cogging torque and the actual power consumption. A lumped parameters model (Fig. 4) is used for the transient thermal computations considering the electromagnetic losses computed during the electromagnetic simulation. The windings maximum operating temperature and the magnets maximum operative temperature are estimated. The electromagnetic properties of the materials of the motor actually depend on the temperature, which in its turn depends on the heat generated by the electromagnetic losses in the materials of the excited motor. Therefore, an iterative process is needed.

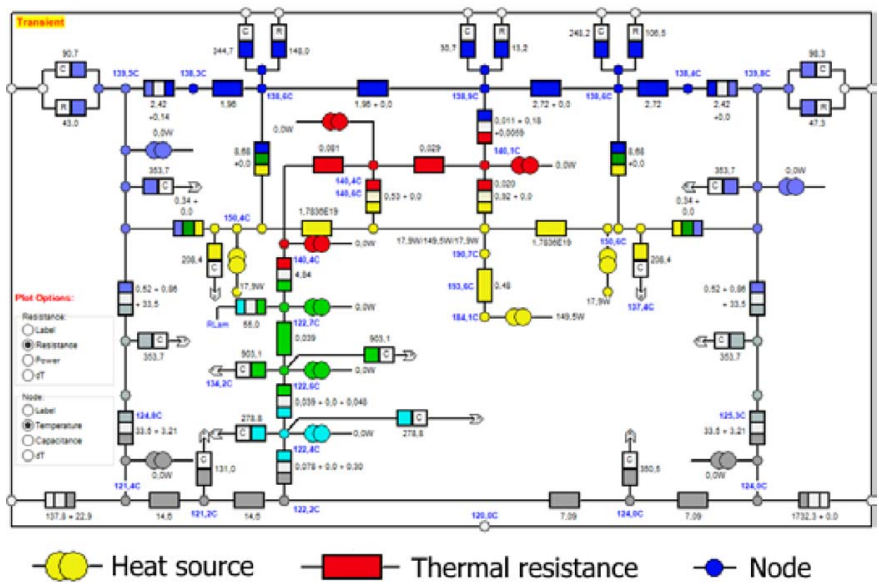

Figure 4. Thermal lumped parameters model (Motor-CAD®).

A two-dimensional FE model developed in ABAQUS ${ }^{\circledR}$ is used to evaluate the stress level in the rotor at the most critical loading conditions $[21,22,23]$. A Python script is used to update the geometry of the parts for a given set of parameters values and to take into account the mechanical and thermal loading of the components resulting from the Motor-CAD® simulations.

\section{Optimisation problem formulation}

The electric motor design problem is formulated as a Multi-Objective (MO) optimisation problem

Page 2 of 6 
$\min _{x \in \mathcal{X}} f(x)$

$g_{i}(\boldsymbol{x}) \leq 0, \quad i=1, \ldots, n_{c}$

where $f(x)$ is the vector of the k objective functions, $x$ the vector of $n$ design variables, $\boldsymbol{X}$ the design variables domain and $\boldsymbol{g}(\mathrm{x})$ the vector of inequality constraints.

In such a problem formulation the concept of optimal solution is not obvious. In general, there is not a single solution. The definition of the optimal solutions in a multi-objective framework has been proposed by Pareto [5]. A Pareto-optimal solution $\boldsymbol{x}_{i}$ is that for which

$$
\begin{aligned}
& \nexists x_{j}: \\
& \left\{\begin{array}{l}
f_{k}\left(x_{j}\right) \leqslant f_{k}\left(x_{i}\right) \quad k=1,2, \ldots n_{o f} \\
\exists l: f_{l}\left(\boldsymbol{x}_{j}\right)<f_{l}\left(\boldsymbol{x}_{i}\right)
\end{array}\right.
\end{aligned}
$$
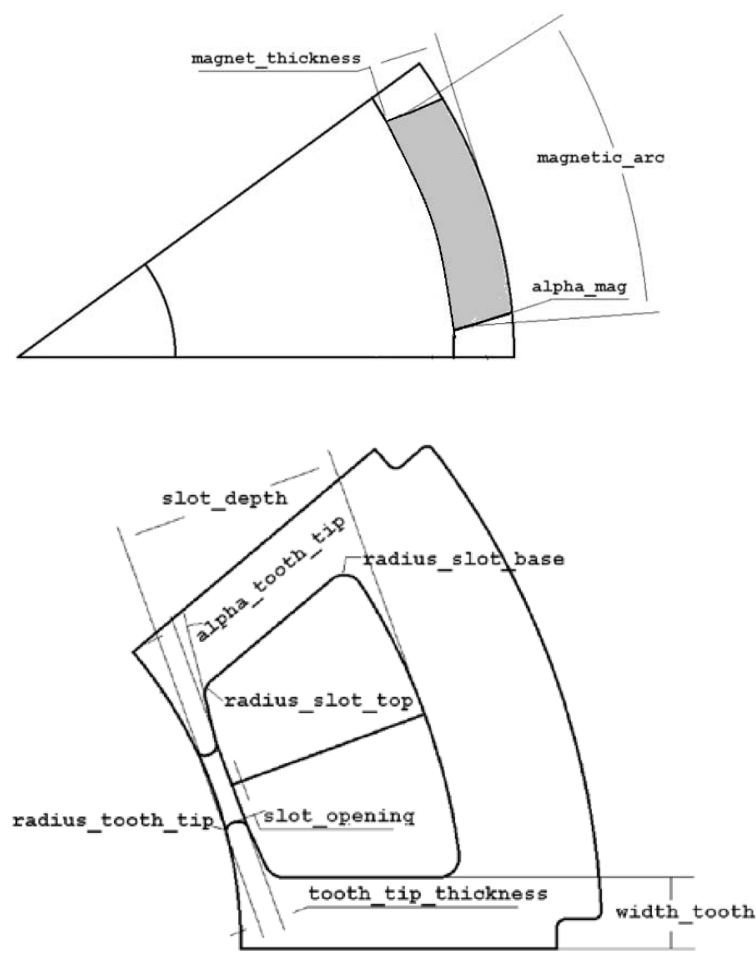

Figure 5. Geometry main design variables, rotor (top), stator (bottom), see Fig. 2 for the electric motor layout.

The set of all of the Pareto-optimal solutions defines the Paretooptimal set. The designer must therefore choose one single Paretooptimal solution. Pareto-optimal solutions are the best obtainable compromise among conflicting objectives.

\section{Design variables}

Fifteen design variables are considered for definition of the stator and rotor geometry, pole pieces and permanent magnets, see Fig. 5 for the definition of the main geometrical design variables.

The parametrised geometry of the motor section is drawn by using the commercial software AutoCAD ${ }^{\circledR}$. Then a VBA (Visual Basic for Application) Excel ${ }^{\circledR}$ code is used to update the drawing parameters on a given set of values and to save the updated geometry so that it could be analysed. A MATLAB ${ }^{\circledR}$ code was developed to import the geometry in Motor-CAD $\AA$, to run the electromagnetic and thermal analyses and to export the results.

\section{Objective functions}

The considered three objective functions are

- $\quad T M$ maximum holding torque (to be maximised), $I_{z z}$ rotor inertia (to be minimised)

- $\quad P$ energy consumption while stalling at $1 \mathrm{Nm}$ (efficiency index, to be minimised).

\section{Constraints functions}

Five geometry constraints have been introduced to avoid non feasible designs (rotor geometry, stator geometry, ..).

Additionally, design constraints that refer to components stress levels, temperature thresholds and maximum cogging torque are applied. They read

$$
\begin{aligned}
& \mathrm{T}_{\text {windings }}<\mathrm{T}_{\mathrm{w} \text { max }} \\
& \mathrm{T}_{\text {magnets }}<\mathrm{T}_{\text {mag max }} \\
& \sigma \text { Von Mises, rotor }<\sigma_{\text {yield }}
\end{aligned}
$$

Cogging Torque $<$ Admissible cogging torque

A Global approximation approach is used to reduce the computation time. The global approximation process is shown in Fig. 6.

Design of Experiment (DOE) [7] approach is applied to define one thousand design solutions to be simulated by means of the physical models (Electromagnetic, Thermal and Structural) to be used as 'training set'. A global approximation model is constructed by means of Neural Networks. The accuracy of the global approximation model is tested in terms of generalisation capability [19], i.e. the errors of the $\mathrm{NN}$ have been computed on a 'validation set' constituted by 100 designs not belonging to the 'training set'. If the accuracy is not 
adequate, the process can be iterated by increasing the number of simulations used to train the $\mathrm{NN}$ and/or by varying the $\mathrm{NN}$ architecture.

Table 1 shows the influence of the training set size on the accuracy of the approximation of the Neural Network. Increasing the size of the training set, the mean absolute percentage error diminishes.

Table 1. NN training. Mean absolute percentage error (MAPE [24]) of the prediction of the $\mathrm{NN}$ for the three objective functions.

\begin{tabular}{c|c|c|c|c} 
Iteration $\mathrm{n}^{\circ}$ & Training set size & $\operatorname{MAPE}(T M)$ & $\operatorname{MAPE}\left(I_{z z}\right)$ & $\operatorname{MAPE}(P)$ \\
\hline \hline 1 & 1000 elements & $0.91 \%$ & $2.01 \%$ & $5.63 \%$ \\
\hline 2 & 2000 elements & $0.16 \%$ & $0.36 \%$ & $0.94 \%$ \\
\hline
\end{tabular}

The NN approximated model is thousands of time faster than the physical models used to train it (120s per design for the physical model, $0.01 \mathrm{~s}$ for the $\mathrm{NN}$ ).

The trained NN has been used to evaluate the performances of a large set of experiments (1E6 different design solutions, see sub-section Design of Experiments). The design constraints have been enforced and the Pareto-optimal solutions have been selected on the basis of Eq. 2 .

The optimisation procedure has been iterated until the mean absolute percentage error (MAPE [24]) of the prediction for each objective function was below $1 \%$ for the design solutions on the Pareto front.

Further details are provided in the following sub-sections.

\section{Design of Experiments}

When the dimensionality of the problem increases, the design variables domain volume increases so fast that the available data become sparse and the amount of data needed in the sampling phase grows exponentially with the dimensionality (curse of dimensionality). Several sampling techniques [8] can be employed in order to define "low discrepancy sequences" having almost the same discrepancy of a uniform grid but using a limited number of samples. Discrepancy is a quantitative metric on how evenly the design variables domain is sampled. A few thousand samples have been used in the solution of the problem on the basis of a Sobol sequence, the design variables have been varied within $\pm 20 \%$ of the reference values.

\section{Global approximation}

The NN architecture is shown in Fig. 7. Artificial neural networks $(\mathrm{NN})$ are a wide class of flexible non-linear regression approximation models. NN were inspired by the data-processing characteristics that are typical of the brain. NN are data-driven self-adaptive methods in that there are reduced a priori assumptions on the NN structure. A multi-layer neural network is able to model a complex non-linear function with an arbitrary degree of accuracy [9]. The proof is given by the universal approximation theorem in $[10,11]$. However, the theorem doesn't state that a single hidden layer is optimum for the generalization properties of the $\mathrm{NN}[12,13,14]$. A feedforward

Page 4 of 6 backpropagating neural network (NN) $[16,17,18]$ with 15 inputs, 6 outputs and 2 hidden layers has been designed (see Fig. 10 for the NN architecture).

\section{Search Method}

The Parameter Space Investigation (PSI) method has been applied $[5,8]$. The feasible and Pareto optimal solutions are determined by applying the definition given in Eq. 2.

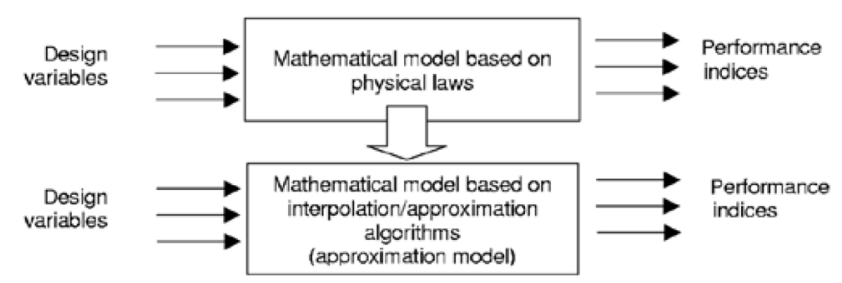

Figure 6. Global approximation procedure.

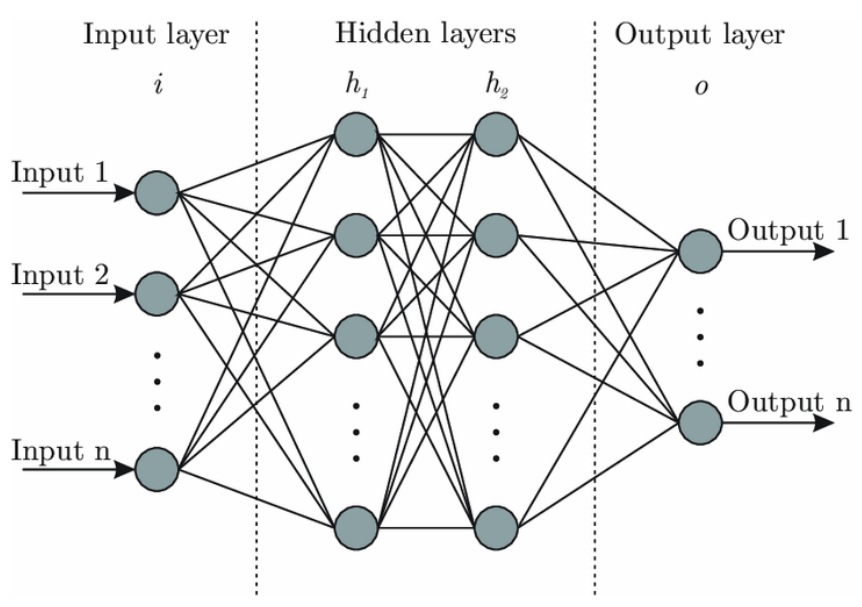

Figure 7. Surrogate model: Neural Network layout.

\section{Optimisation results}

The feasible domain and the Pareto-optimal solutions are shown in Fig. 8 in the objective functions domain. Some Pareto-optimal solutions, highlighted in Fig. 9, are analysed in the following subsections. 


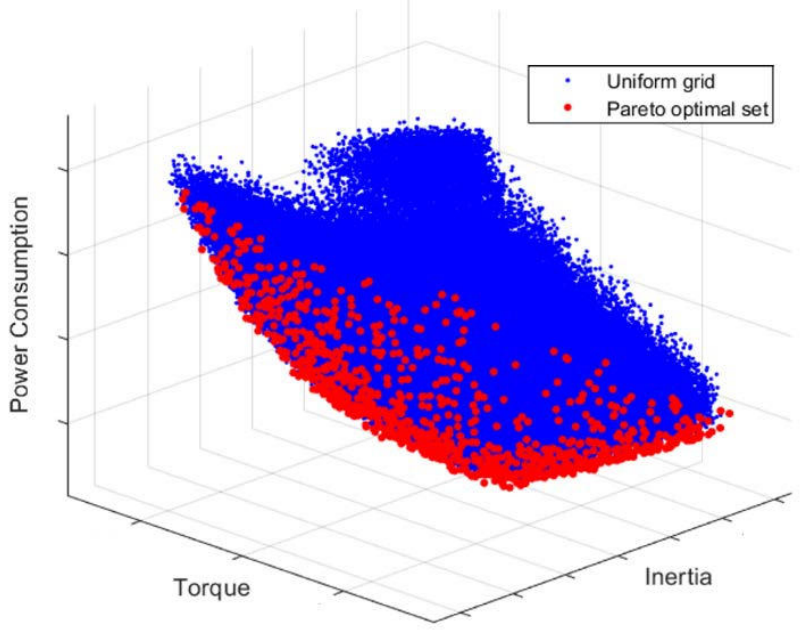

Figure 8. Feasible and optimal (Pareto) designs.

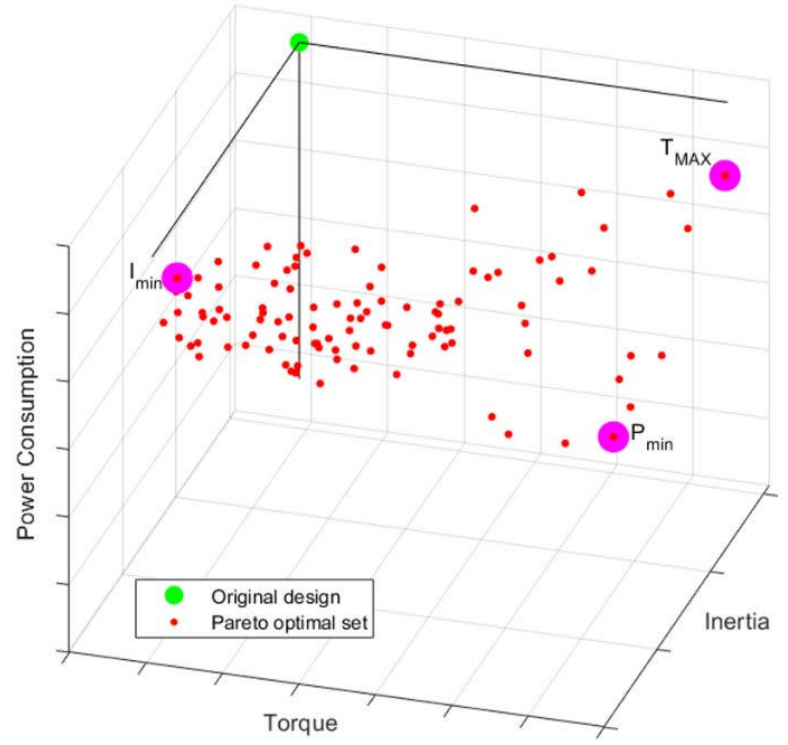

Figure 9. Pareto Optimal solutions.

Solution $\boldsymbol{T}_{\text {MAX }}(+10,3 \%$ Torque, see Fig. 9)

The increase in torque at low speeds is larger than $10 \%$, but a torque drop is shown at intermediate and high speeds. Actually, for a brake caliper actuation system, the most important aspect is responsiveness, therefore the torque at low speeds is the most relevant index of performance.

The rotor inertia is equal to the original one and the power consumption is reduced (1.9\% of power reduction). All the design constraints (cogging torque, maximum stress levels, maximum operative temperature) are satisfied.

Referring to the design variables, the stator section has been widened especially at the tooth tip and stem region. This leads to a reduction in Page 5 of 6 the magnetic saturation of the ferromagnetic material that constitutes the magnetic circuit, allowing for a larger magnetic flux value and thus more torque. The curvature radii at the tooth tip and slot corners are reduced, but are within the feasibility constraints.

The magnet section area is increased, both in width and radial thickness. A bigger magnet produces larger magnetic field thus yielding a greater torque. The extension of the magnet arc has been increased to generate a greater magnetic field. In this optimal design, the magnetic flux needs to flow through a shorter path to get to the adjacent magnet, therefore increasing the magnetic flux value for same winding currents.

\section{Solution $I_{\min }(-34,3 \%$ Inertia, see Fig. 9)}

The rotor inertia is reduced by almost $35 \%$. This is achieved by a $12 \%$ reduction of the stator bore radius. The torque characteristic is very close to the original one across all the speed ranges and the power consumption very close to the original one.

\section{Conclusions}

In this paper, an efficient method for the optimal design of electric motors has been developed. The method is based on the coupling of multi-physical models of the electric motor and global approximation techniques to be integrated into an efficient multi objective optimisation framework.

The ultimate goal is to optimise the shape of the motor components.

Several design variables are considered for the definition of the stator and rotor geometry, pole pieces and permanent magnets. Objective and constraint functions are computed by performing a multi-physical simulation of the electric motor. Three performance indices have been considered in the design process, the starting torque of the motor, the inertia of the rotor and the power consumption. Maximum winding temperature during a braking cycle, maximum Von Mises stress in the rotor at operative conditions and cogging torque ripple are the considered design constraints.

A global approximation approach based on neural networks (feed forward back propagating) has been applied. An initial batch of 1000 experiments has been used to sample the design variables domain through a DOE approach based on a low discrepancy sequence. The $\mathrm{NN}$ has been used to evaluate the performances of a batch of 1 million feasible motor designs whose design variables combinations have been generated on the basis of low discrepancy sequences. The geometry of both stator and rotor has been checked by including manufacturability constraints.

The Pareto-optimal solutions have been selected. The optimisation procedure has been iterated until the mean error on the Pareto-optimal solutions is below $1 \%$.

Improvements up to $10 \%$ on maximum torque, $34 \%$ of rotor inertia reduction and $24 \%$ of power consumption reduction are shown with respect to a reference configuration.

The multi-objective optimisation formulation with the implementation of a global approximation approach has proved to be a robust and efficient approach in designing the electric motor. 


\section{References}

1. SAE International, Information on Electric Brakes, 2019. [Online]. Available at https://doi.org/10.4271/AIR5937

2. Hanselman D., Brushless Permanent Magnet Motor Design. The Writers Collective, 2003.

3. Goss J., Wrobel R., Mellor P., and Staton D., The design of AC permanent magnet motors for electric vehicles: A design methodology, in 2013 International Electric Machines Drives Conference, May 2013, pp. 871-878.

4. Kim S.-H., Alternating current motors. Electric Motor Control, 2017.

5. Mastinu G., Gobbi M., and Miano C., Optimal Design of Complex Mechanical Systems with Applications to Vehicle Engineering. Springer-Verlag Berlin, Heidelberg, 2006.

6. Di Barba P., Multiobjective Shape Design in Electricity and Magnetism, Springer, 2010.

7. Simpson T. W., Peplinski J. D., Koch P. N., and Allen J. K., On the Use of Statistics in Design and the Implications for Deterministic Computer Experiments, Design Theory and Methodology, 1997.

8. Tezuka S., Uniform Random Numbers. Theory and Practice. Springer US, 1995.

9. Haykin S., Neural Networks and Learning Machines. Pearson, 2009.

10. Cybenko G., Approximation by Superpositions of a Sigmoidal Function, Mathematics of Control, Signals, and Systems, vol. 2, pp. 303-314, 1989.

11. Hornik K., Approximation Capabilities of Multilayer Feedforward Networks, Neural Networks, vol. 4, pp. 251-257, 1991.

12. Chester D. L., Why two hidden layers are better than one, in International Joint Conference on Neural Networks, vol. 1, 1990, pp. 265-268.

13. Thomas A. J., Petridis M., Walters S. D., Gheytassi S. M., and Morgan R. E., Two Hidden Layers are Usually Better than One, in Engineering Applications of Neural Networks, G. Boracchi, I. Lazaros, and J. A. L. Chrisina, Eds., 2017.

14. Xu S. and Chen L., A Novel Approach for Determining the Optimal Number of Hidden Layer Neurons for FNNs and Its Application in Data Mining, $5^{\text {th }}$ International Conference on Information Technology and Applications, 2008.

15. Burden F. and Winkler D., Bayesian Regularization of Neural Networks. Artificial Neural Networks. Springer, 2008, vol. Chapter 3.

16. Wortmann T., Genetic evolution vs. function approximation: Benchmarking algorithms for architectural design optimization, Journal of Computational Design and Engineering, 2018.

17. Hagan M. T., Demuth H., and Beale M., Neural Network Design. PWS Publishing Company, 1995.

18. Koziel S., Ciaurri D.E., and Leifsson L., Computational Optimization, Methods and Algorithms. Springer, 2011, ch. 3: Surrogate-Based Methods, pp. 3359.

19. Forrester A. I. J., Sobester A., and Keane A. J., Engineering Design via Surrogate Modelling. A Practical Guide. Wiley, 2008.

20. Rashid M.H., Power Electronics Handbook, ButterworthHeinemann, Ed., 2017.

21. Eliezer Z., Schulz C.J., and Barlow J.W., Friction and wear properties of an epoxy-steel system. Wear, 1978.

22. Dowling N.E., Mechanical Behaviour of Materials. Pearson, 2012. 23. Garg A.C. and Mai Y.W., Failure mechanisms in toughened epoxy resins - A review, Composites Science and Technology, 1988.

24. Armstrong J.S., Collopy F., Error Measures for Generalizing About Forecasting Methods: Empirical Comparisons, International Journal of Forecasting, 8, pp. 69-80, 1992.

25. Statnikov R., Statnikov A., The Parameter Space Investigation Method Toolkit, Boston/London: Artech House, 2011.

Page 6 of 6
26. Lazzari M., Miotto A., Tenconi A., Vaschetto S., Analytical prediction of eddy current losses in retaining sleeves for surface mounted PM synchronous machines, XIX International Conference on Electrical Machines - ICEM 2010, IEEE, 2010.

27. Skaar S.E., Krøvel Ø., Nilssen R. Distribution, coil-span and winding factors for PM machines with concentrated windings, IEEE International Electric Machines and Drives Conference, IEMDC'03, 2003.

\section{Contact Information}

Prof. Gianpiero Mastinu, Politecnico di Milano, Department of Mechanical Engineering, Via la Masa 1, 20156 Milano. E-mail: gianpiero.mastinu@polimi.it. Telephone: +39.02.2399.8289.

\section{Acknowledgments}

Lombardy Region Government has partially funded the research with POR/FESR INPROVES grant (ID 241363).

$\begin{array}{ll}\text { Abbreviations } & \\ \text { EMA } & \text { Electromechanical actuator } \\ \text { BLDC } & \text { Brushless DC electric motor } \\ \text { PM } & \text { Permanent magnets } \\ \text { NN } & \text { Neural Network } \\ \text { MO } & \text { Multi-objective } \\ \text { DOE } & \text { Design of Experiments } \\ \text { MAPE } & \text { Mean absolute percentage error }\end{array}$

